\title{
Facile and efficient synthesis of $4^{\prime}$-thioxo-1',3,3',4,4',6'-hexahydro- $1 H, 2^{\prime} H$-spiro(naphthalene-2,5'-pyrimidin)-1-ones in a three-component Mannich-type reaction
}

\author{
Tadeusz S. Jagodziński* and Sławomir Westerlich \\ Institute of Chemistry and Environmental Protection, West Pomerania University of Technology, \\ Aleja Piastów 42, 71-065 Szczecin, Poland \\ E-mail: jagszcz@zut.edu.pl
}

\begin{abstract}
Spirohexahydropyrimidines were obtained in the one-pot three-component reaction of the tetralone-derived thioamides, amines and formaldehyde with alcoholic hydrogen chloride as the catalyst.
\end{abstract}

Keywords: Mannich reaction, thioamides, spirohexahydropyrimidines

\section{Introduction}

Although thioamides are known almost since the very dawn of organic chemistry, their synthetic applicability gained a wide recognition only a few decades ago. High reactivity of the thioamide group towards both electro- and nucleophilic agents, often in conjunction with that of other reactive centers in the molecule, made thioamides handy building blocks of particular importance in the synthesis of heterocyclic systems by inter- and intramolecular cyclization. ${ }^{1-5}$ Saturated or partially saturated heterocyclic compounds with a thioamide group were found to be of interest as potential ionic liquid media. ${ }^{6}$ Bogdanowicz-Szwed and co-workers reported the base-catalyzed reaction of $\beta$-ketothioamides with $(E)$ - $\beta$-nitrostyrenes which yielded spiro(indane$1,3^{\prime}$-thiophenes). ${ }^{7,8}$

On the other hand, the hexahydropyrimidine fragment is present in several natural products and in pharmaceutical agents of various biological activity. ${ }^{9-12}$ Some derivatives of hexahydropyrimidine are used as stabilizers of polymers. ${ }^{13}$ Owing to their facile cleavage in mild acidic medium, hexahydopyrimidines have been employed in organic synthesis as protective groups in the selective acylation and addition of 1,3-diamines. ${ }^{18}$

The classic synthetic route leading to this type of heterocyclic compounds involves the condensation of substituted propane-1,3-diamines with aldehydes and ketones. ${ }^{14-17}$ Liang and coworkers recently synthesized 1,3-diaryl-5-spirohexahydropyrimidines via the proline-catalyzed 
one-pot condensation of anilines, formaldehyde, and cyclohexanones in the Mannich-type reaction, ${ }^{19}$ which as a powerful $\mathrm{C}-\mathrm{C}$ bond formation process is widely applied to the preparation of diverse aminoalkyl derivatives. ${ }^{20-23}$

In recent years multicomponent reactions (MCRs) have attracted the attention of organic chemists because of their numerous advantages over conventional multistep syntheses. ${ }^{24-26}$ Thus, Mukhopadhyay and co-workers ${ }^{27}$ reported a multicomponent synthesis of 1,3diarylhexahydropyrimidines in the one-pot reaction of 1,3-dicarbonyl compounds, aromatic amines and formaldehyde catalyzed by iron(III) chloride. T. B. Shah and co-workers ${ }^{28}$ described their results of the synthesis and in vitro biological screening of a series of $N$-Mannich bases obtained from 3,4-dihydropyrimidine-2-(1H)-thiones; those with morpholine as the heterocyclic secondary amino component showed a promising activity against both bacterial and fungal microorganisms. $^{28}$

In our earlier papers we have described the reactions of intramolecular cyclization of $N$ allylthioamides derived from cyclic ketones and $\beta$-diketones, ${ }^{29}$ the reactions of $\beta$-keto thioamides with carboxylic esters, ${ }^{30}$ as well as the reactions of $\beta$-ketothioamides with $\alpha, \beta$-unsaturated aldehydes. $^{31}$

\section{Results and Discussion}

Continuing our studies on the application of $\beta$-keto thioamides to the synthesis of heterocyclic compounds we report in the present paper a facile and efficient synthesis of $4^{\prime}$-thioxo$1^{\prime}, 3,3^{\prime}, 4,4^{\prime}, 6^{\prime}$-hexahydro- $1 H, 2^{\prime} H$-spiro(naphthalene-2,5'-pyrimidin)-1-ones in a three-component Mannich-type reaction.

Neither room nor elevated (approx. $40^{\circ} \mathrm{C}$ ) temperature conditions were found effective when the reaction of 2-tetralone derivatives $\mathbf{1}$ with secondary amines (dimethylamine, morpholine) was carried out according to the classic Mannich procedure. However, when primary aliphatic amines 2 were used under analogous conditions at room or slightly elevated (approx. $40{ }^{\circ} \mathrm{C}$ ) temperatures, spiroheterocyclic compounds 3-10 were readily formed in good yields (Scheme 1), although aromatic amines failed to react as shown in the experiments with the 2-tetralonederived thioamides $\mathbf{1}$ or 1,3-indanedione 11. Methanol, ethanol or THF containing a catalytic amount of hydrochloric acid served as the reaction medium. The failure with secondary and aromatic amines may be due to steric hindrance, though no experiments have been made to substantiate that assumption.

In the earlier studies on the three-component reactions of amines and formaldehyde with $\beta$ diketones or $\beta$-keto esters, double aminomethylation was observed to occur at the $\alpha$ carbon atom. ${ }^{27}$ Six molecules of reactants were involved and six new covalent bonds were formed in this one-pot three-component reaction; the highest yields of hexahydropyrimidines were obtained when the 1,3-carbonyl compound : amine : formaldehyde molar ratio was $1: 2: 3$. An analogous 
reaction course was reported in the case of the proline-catalyzed aminomethylation of cyclohexanone. $^{19}$<smiles>O=C1c2ccccc2CCC1C(=S)NI</smiles>

1<smiles>[R16]N</smiles>

2

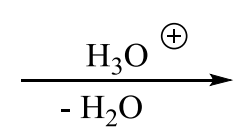<smiles>[R]N1CCCCCC2(CCc3ccccc3C2=O)C(=S)N([R])C1</smiles>

3-10

3: $\mathrm{R}^{1}=\mathrm{Ph}, \mathrm{R}^{2}=n-\operatorname{Pr}(81)$

4: $\mathrm{R}^{1}=\mathrm{Me}, \mathrm{R}^{2}=\mathrm{Bn}(83)$

7: $\mathrm{R}^{1}=\mathrm{Ph}, \mathrm{R}^{2}=i-\mathrm{Bu}(72)$

5: $\mathrm{R}^{1}=\mathrm{Ph}, \mathrm{R}^{2}=\mathrm{Bn}(78)$

8: $R^{1}, R^{2}=\mathrm{Me}(82)$

6: $\mathrm{R}^{1}=\mathrm{Me}, \mathrm{R}^{2}=i$ - $\mathrm{Bu}(75)$

9: $\mathrm{R}^{1}=\mathrm{Ph}, \mathrm{R}^{2}=\mathrm{Me}$ (84)

10: $\mathrm{R}^{1}=\mathrm{Ph}, \mathrm{R}^{2}=3-\mathrm{PyCH}_{2}(79)$

Scheme 1. Synthesis of (+/-)-1',3'-disubstituted-4'-thioxo-1',3,3',4,4',6'-hexahydro- $1 H, 2^{\prime} H$ spiro(naphthalene-2,5'-pyrimidin)-1-ones.

Our results indicate that the thioamide-group nitrogen atom takes part in the formation of the pyrimidine ring. Furthermore, in comparison with similar reactions of ketones ${ }^{19,27}$ the molar ratio of reagents is much different, in our case. In the preparative procedures both the amine and formaldehyde were used in an excess in order to optimize the yield of the hexahydropyrimidines. In contrast to the reported reactions of (2-substituted thiocarbamoyl)malonic acid diethyl esters ${ }^{32}$ no advance generation of the thioamide anion is required.

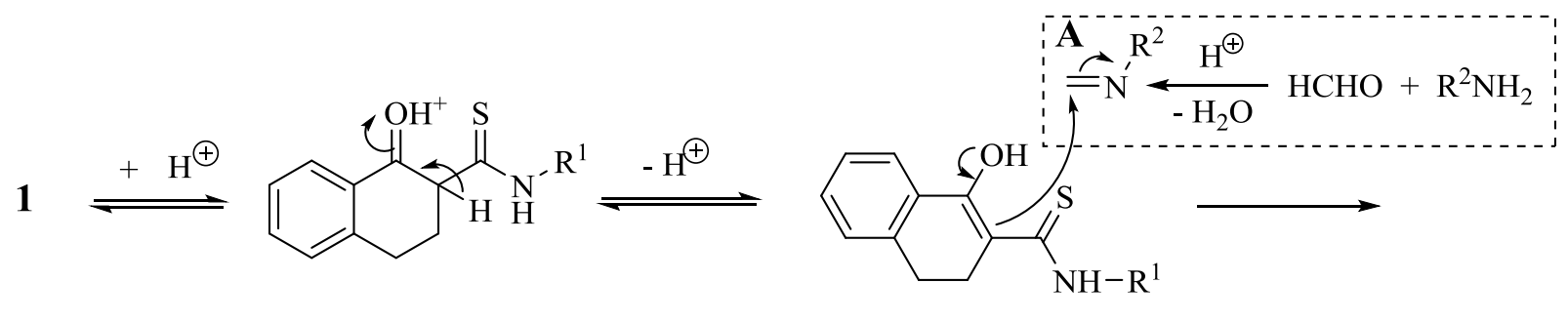

B

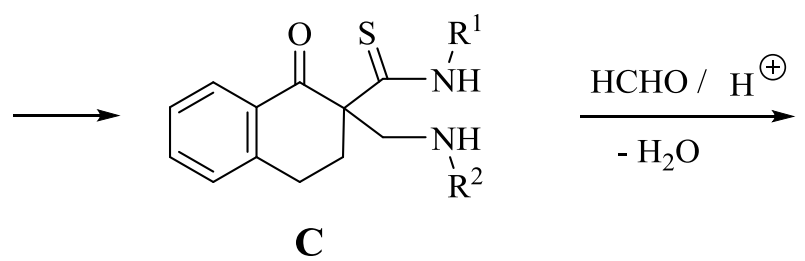<smiles>[R]N1CN([R])C(=S)C2(CCc3ccccc3C2=O)C1</smiles>

3 - 10

Scheme 2. Proposed mechanism for the reaction. 
The suggested mechanistic explanation of this reaction assumes that the electrophilic imine $\mathbf{A}$ attacks the enol form $\mathbf{B}$ of the thioamide at both its sides to produce two enantiomeric amino thioamides $\mathbf{C}$ which subsequently condense with another molecule of formaldehyde to yield ultimately the racemic mixtures of $(R, S)$-1',3'-disubstituted-4'-thioxo-1',3,3',4,4',6'-hexahydro$1 H, 2^{\prime} H$-spiro(naphthalene-2,5'-pyrimidin)-1-ones 3-10 (Scheme 2). The reaction yield was roughly independent of the substituent attached to the thioamide nitrogen atom.

Thioamides derived from cyclic $\beta$-ketodiketones were found to react similarly. For example, 1,3-dioxoindane-2-carbothioic $N$-methylamide (11) with benzylamine yielded the spirocyclic compound 12 (Scheme 3).

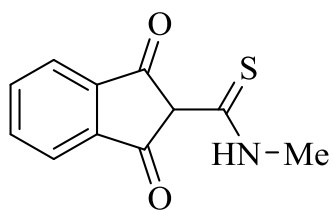

11

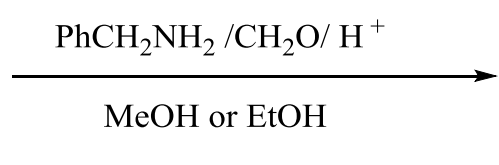

$\mathrm{MeOH}$ or $\mathrm{EtOH}$

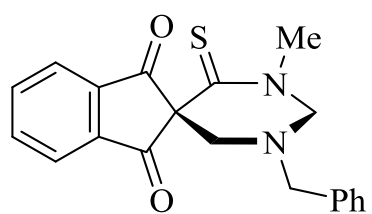

12

Scheme 3. Synthesis of $1^{\prime}$-benzyl-3'-methyl-4'-thioxo-2', $3^{\prime}, 4^{\prime}, 6^{\prime}$-tetrahydro-1'H-spiro(indene2,5'-pyrimidine)-1,3-dione.

In our preliminary experiments on the reactions of linear $\beta$-ketothiomides, 3-oxo-3, $N$ diphenylthiopropionamide (13) readily reacted with benzylamine and formaldehyde to give in high yield $(88 \%)$ the racemic hexahydropyrimidines (14). Detailed results will be shortly published in full.

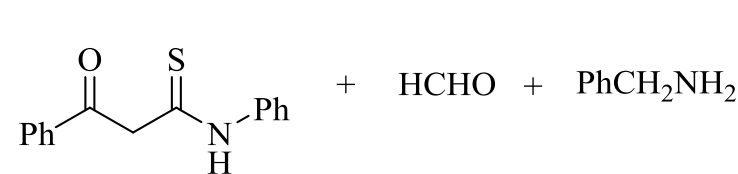

13
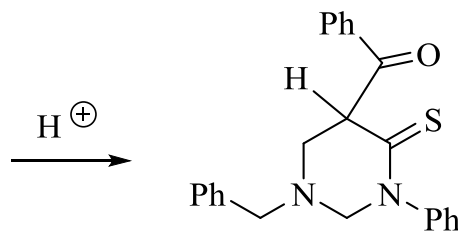

14

Scheme 4. Synthesis of (1-benzyl-3-phenyl-4-thioxohexahydropyrimidin-5-yl)phenylmethanone.

\section{Conclusions}

Aminomethylation of the thioamides with an acidic hydrogen atom at the $\alpha$ carbon atom has to be considered as a general reaction. Its substrates are easily available and the products are formed at room or slightly elevated temperatures in usually high yields. A proper choice of the starting thioamides and amines makes it possible to use this reaction in order to synthesize 
several new and potentially useful hexahydropyrimidine derivatives, for example biologically active compounds.

\section{Experimental Section}

General. Melting points were determined in a Boëtius hot-stage apparatus and are uncorrected. The NMR spectra were recorded with a Bruker DPX (400 MHz and $100 \mathrm{MHz}$ for ${ }^{1} \mathrm{H}$ NMR and ${ }^{13} \mathrm{C}$ NMR, respectively) spectrometer in deuteriochloroform with tetramethysilane as internal standard. The IR spectra were taken with Specord M80 instruments in potassium bromide pellets or nujol. Purity and molecular mass determinations were carried out by gas chromatographymass spectrometry (GC/MS) with a Hewlett-Packard instrument model HP 6890 equipped with a mass detector HP 5973 (8 only, other compounds decomposed); the analytical procedure was developed for a $30-\mathrm{m}$ long capillary column, $0.2 \mathrm{~mm}$ in diameter, with methylsiloxane modified with phenyl groups $(5 \% \mathrm{Ph}, \mathrm{Me}$ siloxane) in the $0.25 \mu \mathrm{m}$ thick active phase layer. Elemental analyses were performed on EuroEA 3000series Euro Vector CHNS-O. All compounds gave satisfactory elemental analysis results $(\mathrm{C}, \mathrm{H}, \mathrm{N}, \mathrm{S})$. The starting thioamides $\mathbf{1}$ were prepared as described earlier. ${ }^{8,33}$

\section{Synthesis of compounds 3-10, 14}

A mixture of thioamide 1 or $\mathbf{1 3}(1 \mathrm{mmol})$, alkylamine $\mathbf{2}$ (3 mmol), formaldehyde (6 mmol, 37\% aqueous solution) in $20 \mathrm{~mL}$ of $\mathrm{EtOH}$ or $\mathrm{MeOH}$ containing few drops of conc. $\mathrm{HCl}$ was stirred at room temperature for $1 \mathrm{~h}$ and then left overnight. The reaction progress was monitored by TLC (silufol /chloroform or hexane : ethyl acetate 1:1). After the completion of the reaction (1-24 hours), the solvent was removed by evaporation. An aqueous sodium carbonate solution (5\%) was added and the resulting mixture was extracted with ethyl acetate. The extract was washed with water, dried with $\mathrm{MgSO}_{4}$, and passed under reduced pressure through a short column (8 $1.5 \mathrm{~cm}$ ) packed with $\mathrm{Al}_{2} \mathrm{O}_{3}$ (Brockmann I, neutral, standard) using ethyl acetate as the eluent; reduced pressure was applied to make this operation less time-consuming. Upon evaporation of the solvent, the residue was recrystallized from EtOH or $\mathrm{n}_{-} \mathrm{C}_{6} \mathrm{H}_{14} / \mathrm{EtOH}$. Samples used in elemental analyses were chromatographed in a silica gel packed column with chloroform or a hexane/ethyl acetate $(1: 1$ or $2: 1)$ mixture as the eluent and finally recrystallized again from an appropriate solvent.

3'-Phenyl-1'-propyl-4'-thioxo-2',3,3',4,4',6'-hexahydro-1H,1'H-spiro(naphthalene-2,5' pyrimidin)-1-one (3) White powder, yield 81\%; m.p. 69-71 ${ }^{\circ} \mathrm{C}\left(\mathrm{n}-\mathrm{C}_{6} \mathrm{H}_{14} / \mathrm{EtOH}\right)$; IR $\left(v_{\max }, \mathrm{cm}^{-1}\right)$ : $1678(\mathrm{C}=\mathrm{O}) .{ }^{1} \mathrm{H} \mathrm{NMR}\left(\mathrm{CDCl}_{3}\right): \delta_{\mathrm{H}} 0.91\left(3 \mathrm{H}, \mathrm{t}, J 7.3 \mathrm{~Hz}, \mathrm{CH}_{3}\right), 1.50\left(2 \mathrm{H}, \mathrm{q}, J 7.3 \mathrm{~Hz}, \mathrm{CH}_{3} \mathrm{CH}_{2}\right)$, 2.32-2.44 (2H, m, $\left.\mathrm{CH}_{2} \mathrm{C} \underline{\mathrm{HHC}}, \mathrm{NC} \underline{\mathrm{HH}}\right), 2.56\left(2 \mathrm{H}, \mathrm{t}, J\right.$ 7.1 Hz, NC$\left.\underline{\mathrm{H}}_{2} \mathrm{CH}_{2}\right), 2.95-3.07(1 \mathrm{H}, \mathrm{m}$,

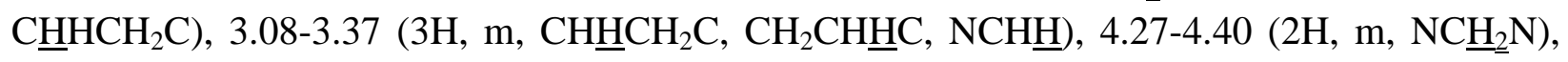
7.09-7.19 (1H, m, Ph), 7.20-7.28 (1H, m, Ph), 7.29-7.41 (3H, m, Ph), 7.43-7.53 (3H, m, Ph), 8.07 $(1 \mathrm{H}, \mathrm{d}, J 7.8 \mathrm{~Hz}, \mathrm{Ph}) .{ }^{13} \mathrm{C} \mathrm{NMR}\left(\mathrm{CDCl}_{3}\right): \delta_{\mathrm{C}} 11.6\left(\mathrm{q}, \mathrm{CH}_{3}\right), 20.2\left(\mathrm{t}, \mathrm{CH}_{3} \mathrm{CH}_{2} \mathrm{CH}_{2}\right), 25.4((\mathrm{t}$, 


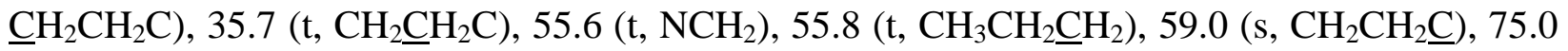
$\left(\mathrm{t}, \mathrm{NCH}_{2} \mathrm{~N}\right), 127.0,127.1,128.3,128.3,128.6,129.9,132.2,133.6,142.8,144.7$ (12C, Ph) 196.7 (s, C=O), 202.8 (s, C=S). Anal. Calcd. for $\mathrm{C}_{22} \mathrm{H}_{24} \mathrm{~N}_{2} \mathrm{OS}$ (364.50): C, 72.49; H, 6.64; N, 7.69; S, 8.80. Found: C, 72.40; H, 7.03; N, 7.56; S, 8.37\%.

1'-Benzyl-3'-methyl-4' -thioxo-2',3,3',4,4',6'-hexahydro-1H,1' $H$-spiro(naphthalene-2,5'pyrimidin)-1-one (4). Yellowish powder, yield 83\%, mp 93-95 ${ }^{\circ} \mathrm{C}(\mathrm{EtOH})$; IR $\left(v_{\max }, \mathrm{cm}^{-1}\right): 1678$ $(\mathrm{C}=\mathrm{O}) .{ }^{1} \mathrm{H}$ NMR $\left(\mathrm{CDCl}_{3}\right): \delta_{\mathrm{H}} 2.28\left(1 \mathrm{H}, \mathrm{dt}, J\right.$ 13.7, $5.0 \mathrm{~Hz}, \mathrm{CH}_{2} \mathrm{CH}$ $\left.\mathrm{C}_{\mathbf{H}} \mathrm{HCH}_{2} \mathrm{C}\right), 2.93(1 \mathrm{H}, \mathrm{d}, J 12.7 \mathrm{~Hz}, \mathrm{NC} \underline{\mathrm{H}}), 2.97-3.11$ (2H, m, NCH$\left.\underline{H}, \mathrm{CH}^{\mathrm{HCH}}{ }_{2} \mathrm{C}\right), 3.12-3.22$ $\left(1 \mathrm{H}, \mathrm{m}, \mathrm{CH}_{2} \mathrm{CH} \underline{\mathrm{HC}}\right), 3.40\left(3 \mathrm{H}, \mathrm{s}, \mathrm{CH}_{3}\right), 3.60(1 \mathrm{H}, \mathrm{d}, J=13.1 \mathrm{~Hz}, \mathrm{C} \underline{H} H \mathrm{Ph}), 3.76(1 \mathrm{H}, \mathrm{d}, J 13.1$

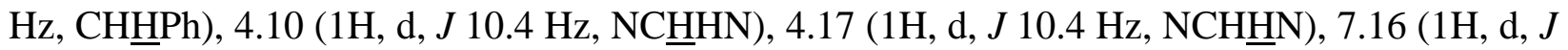
$7.6 \mathrm{~Hz}, \mathrm{Ph}), 7.21-7.35(6 \mathrm{H}, \mathrm{m}, \mathrm{Ph}), 7.39-7.47(1 \mathrm{H}, \mathrm{m}, \mathrm{Ph}), 8.03(1 \mathrm{H}, \mathrm{d}, J 7.9 \mathrm{~Hz}, \mathrm{Ph}) .{ }^{13} \mathrm{C} \mathrm{NMR}$ $\left(\mathrm{CDCl}_{3}\right): \delta_{\mathrm{C}} 25.2\left(\mathrm{t}, \underline{\mathrm{CH}_{2}} \mathrm{CH}_{2} \mathrm{C}\right), 35.2\left(\mathrm{t}, \mathrm{CH}_{2} \underline{\mathrm{CH}}_{2} \mathrm{C}\right), 40.9\left(\mathrm{q}, \mathrm{CH}_{3}\right), 54.7\left(\mathrm{q}, \mathrm{CH}_{3}\right), 58.5(\mathrm{t}$, $\left.\underline{\mathrm{CH}}_{2} \mathrm{Ph}\right), 59.6\left(\mathrm{~s}, \mathrm{CH}_{2} \mathrm{CH}_{2} \underline{\mathrm{C}}\right), 73.1\left(\mathrm{t}, \mathrm{NCH}_{2} \mathrm{~N}\right), 126.8,127.8,128.3,128.5,128.6,128.7,132.3$, 133.5, 136.3, 142.8 (12C, Ph), 196.6 (s, C=O), 200.2 (s, C=S). Anal. Calcd. for $\mathrm{C}_{21} \mathrm{H}_{22} \mathrm{~N}_{2} \mathrm{OS}$ (350.48): C, 71.97; H, 6.33; N, 7.99; S, 9.15. Found: C, 72.04; H, 5.54; N, 7.99; S, 9.20\%.

1'-Benzyl-3'-phenyl-4'-thioxo-2',3,3',4,4',6'-hexahydro-1H,1'H-spiro(naphthalene-2,5' pyrimidin)-1-one (5). Yellowish powder, yield 78\%, mp 143-146 ${ }^{\circ} \mathrm{C}(\mathrm{EtOH}) ; \mathrm{IR}\left(v_{\max }, \mathrm{cm}^{-1}\right)$ : $1672(\mathrm{C}=\mathrm{O}) .{ }^{1} \mathrm{H}$ NMR $\left(\mathrm{CDCl}_{3}\right): \delta_{\mathrm{H}} 2.32-2.43\left(1 \mathrm{H}, \mathrm{m}, \mathrm{CH}_{2} \mathrm{C} \underline{\mathrm{HHC}}\right), 2.72-2.85(1 \mathrm{H}, \mathrm{m}$, $\left.\mathrm{C}_{\underline{H} H C H} \mathrm{C}\right), 3.03-3.17$ (2H, m, $\left.\mathrm{CH}_{\underline{H C H}} \mathrm{C}, \mathrm{NC} \underline{H} \mathrm{H}\right), 3.24$ (1H, d, J 13.2 Hz, NCH$\left.\underline{H}\right), 3.26-3.37$ (1H, m, $\left.\mathrm{CH}_{2} \mathrm{CH} \underline{\mathrm{HC}}\right), 3.71(1 \mathrm{H}, \mathrm{d}, J 13.2 \mathrm{~Hz}, \mathrm{C} \underline{\mathrm{H}} \mathrm{HP}), 3.86(1 \mathrm{H}, \mathrm{d}, J 13.2 \mathrm{~Hz}, \mathrm{CH} \underline{\mathrm{HPh}}), 4.35$ $(1 \mathrm{H}, \mathrm{d}, J 10.6 \mathrm{~Hz}, \mathrm{NC} \underline{\mathrm{HHN}}), 4.42(1 \mathrm{H}, \mathrm{d}, J 10.6 \mathrm{~Hz}, \mathrm{NCH} \underline{\mathrm{HN}}), 7.18(1 \mathrm{H}, \mathrm{d}, J 7.6 \mathrm{~Hz}, \mathrm{Ph}), 7.21-$ $7.40(9 \mathrm{H}, \mathrm{m}, \mathrm{Ph}), 7.41-7.50(3 \mathrm{H}, \mathrm{m}, \mathrm{Ph}), 8.05(1 \mathrm{H}, \mathrm{d}, J 7.8 \mathrm{~Hz}, \mathrm{Ph}) .{ }^{13} \mathrm{C} \mathrm{NMR}\left(\mathrm{CDCl}_{3}\right): \delta_{\mathrm{C}} 25.2$ (t, $\left.\underline{\mathrm{CH}_{2}} \mathrm{CH}_{2} \mathrm{C}\right), 35.5\left(\mathrm{t}, \mathrm{CH}_{2} \underline{\mathrm{CH}}_{2} \mathrm{C}\right), 55.0\left(\mathrm{t}, \mathrm{NCH}_{2}\right), 58.3\left(\mathrm{t}, \underline{\mathrm{CH}_{2}} \mathrm{Ph}\right), 59.3\left(\mathrm{~s}, \mathrm{CH}_{2} \mathrm{CH}_{2} \mathrm{C}\right), 74.7$ (t, $\left.\mathrm{NCH}_{2} \mathrm{~N}\right), 126.9,127.1,127.8,128.3,128.4,128.5,128.6,128.7,129.9,132.2,133.5,136.4$, 142.8, 144.5 (18C, Ph), 196.6 (s, C=O), 203.0 (s, C=S); Anal. Calcd. for $\mathrm{C}_{26} \mathrm{H}_{24} \mathrm{~N}_{2} \mathrm{OS}$ (412.55): C, 75.70; H, 5.86; N, 6.79; S, 7.77. Found: C, 75.31; H, 5.95; N, 6.82; S, 7.66\%.

1'-Isobutyl-3'-methyl-4' -thioxo-2',3,3',4,4', ,6'-hexahydro-1H, ' $^{\prime} H$-spiro(naphthalene-2,5' pyrimidin)-1-one (6). White powder, yield 75\%, mp 114-116 ${ }^{\circ} \mathrm{C}$; IR $\left(v_{\max }, \mathrm{cm}^{-1}\right)$ : $1676(\mathrm{C}=\mathrm{O})$. ${ }^{1} \mathrm{H}$ NMR $\left(\mathrm{CDCl}_{3}\right): \delta_{\mathrm{H}} 0.89\left(6 \mathrm{H}, \mathrm{q}, J 6.6 \mathrm{~Hz}, 2 \mathrm{CH}_{3}\right), 1.64-1.77\left(1 \mathrm{H}, \mathrm{m}, \mathrm{C} \underline{\mathrm{H}}\left(\mathrm{CH}_{3}\right)_{2}\right), 2.23(2 \mathrm{H}, \mathrm{d}, J$ $\left.7.4 \mathrm{~Hz}, \underline{\mathrm{C}}_{2} \mathrm{CH}\right), 2.27-2.36$ (1H, m, $\left.\mathrm{CH}_{2} \mathrm{C} \underline{\mathrm{HHC}}\right), 2.88-3.04$ (3H, m, $\left.\mathrm{CHHCH}_{2} \mathrm{C}, \mathrm{NC} \underline{\mathrm{H}}\right), 3.09-$ $3.25\left(2 \mathrm{H}, \mathrm{m}, \mathrm{CH}_{2} \mathrm{CH} \underline{\mathrm{HC}}, \mathrm{NCH} \underline{\mathrm{H}}\right), 3.42\left(3 \mathrm{H}, \mathrm{s}, \mathrm{CH}_{3}\right), 4.03(1 \mathrm{H}, \mathrm{d}, J 10.4 \mathrm{~Hz}, \mathrm{NC} \underline{H} \mathrm{HN}), 4.11$ $(1 \mathrm{H}, \mathrm{d}, J 10.4 \mathrm{~Hz}, \mathrm{NCH} \underline{\mathrm{HN}}), 7.23(1 \mathrm{H}, \mathrm{d}, J 7.6 \mathrm{~Hz}, \mathrm{Ph}), 7.31(1 \mathrm{H}, \mathrm{t}, J 7.4 \mathrm{~Hz}, \mathrm{Ph}), 7.47$ (1H, td, $J$ 7.4, $1.4 \mathrm{~Hz}, \mathrm{Ph}), 8.04(1 \mathrm{H}, \mathrm{dd}, J 7.8,1.21 \mathrm{~Hz}, \mathrm{Ph}) .{ }^{13} \mathrm{C} \mathrm{NMR}\left(\mathrm{CDCl}_{3}\right): \delta_{\mathrm{C}} 20.5\left(\mathrm{q}, \mathrm{CH}_{3}\right), 20.6(\mathrm{q}$, $\left.\mathrm{CH}_{3}\right), 25.4\left(\mathrm{t}, \underline{\mathrm{CH}}_{2} \mathrm{CH}_{2} \mathrm{C}\right), 25.8\left(\mathrm{~d}, \underline{\mathrm{CH}}\left(\mathrm{CH}_{3}\right)_{2}\right), 35.1\left(\mathrm{t}, \mathrm{CH}_{2} \underline{C H}_{2} \mathrm{C}\right), 40.8$ (q, N-CH$\left.)_{3}\right), 55.3$ (t, $\left.\mathrm{NCH}_{2}\right), 59.6\left(\mathrm{~s}, \mathrm{CH}_{2} \mathrm{CH}_{2} \mathrm{C}\right), 62.2\left(\mathrm{t}, \mathrm{CH}_{2} \mathrm{CH}\left(\mathrm{CH}_{3}\right)_{2}\right), 73.8\left(\mathrm{t}, \mathrm{NCH}_{2} \mathrm{~N}\right), 126.9,128.3,128.5$, 132.3, 133.5, 142.7 (6C, Ph), 196.5 (s, C=O), 199.9 (s, C=S). Anal. Calcd. For $\mathrm{C}_{18} \mathrm{H}_{24} \mathrm{~N}_{2} \mathrm{OS}$ (316.46): C, 68.32; H, 7.64; N, 8.85; S, 10.13. Found: C, 68.01; H, 7.79; N, 8.83; S, $10.09 \%$.

1'-Isobutyl-3'-phenyl-4' -thioxo-2',3,3',4,4',6' 'hexahydro- $1 H$, 1' $H$-spiro(naphthalene-2,5'-

pyrimidin)-1-one (7). White powder, yield 72\%, mp 86-88 ${ }^{\circ} \mathrm{C}\left(\mathrm{n}-\mathrm{C}_{6} \mathrm{H}_{14} / \mathrm{EtOH}\right) . \mathrm{IR}\left(v_{\max }, \mathrm{cm}^{-1}\right)$ : $1676(\mathrm{C}=\mathrm{O}) .{ }^{1} \mathrm{H}$ NMR $\left(\mathrm{CDCl}_{3}\right): \delta_{\mathrm{H}} 0.91\left(6 \mathrm{H}, \mathrm{q}, J 6.6 \mathrm{~Hz}, 2 \mathrm{CH}_{3}\right), 1.63-1.83\left(1 \mathrm{H}, \mathrm{m}, \mathrm{CH}\left(\mathrm{CH}_{3}\right)_{2}\right)$, 
2.35 (2H, dd, J 7.2, $\left.2.8 \mathrm{~Hz}, \mathrm{C}_{2} \underline{\mathrm{CH}}\right), 2.37-2.46\left(1 \mathrm{H}, \mathrm{m}, \mathrm{CH}_{2} \mathrm{C} \underline{\mathrm{H}} \mathrm{HC}\right), 2.96-3.06$ (1H, m, $\mathrm{C}_{\underline{H} H C H} \mathrm{C}$ ), 3.09-3.27 (3H, m, $\left.\mathrm{CH}_{1} \mathrm{HCH}_{2} \mathrm{C}, \mathrm{NC} \underline{\mathrm{HH}}\right), 3.29-3.38$ (1H, m, $\left.\mathrm{CH}_{2} \mathrm{CH} \underline{\mathrm{HC}}\right), 4.29$ (1H, d, $J 10.6 \mathrm{~Hz}, \mathrm{NC} \underline{H} \mathrm{HN}), 4.32(1 \mathrm{H}, \mathrm{d}, J 10.6 \mathrm{~Hz}, \mathrm{NCH} \underline{\mathrm{HN}}), 7.24(1 \mathrm{H}, \mathrm{d}, J 7.7 \mathrm{~Hz}, \mathrm{Ph}), 7.29-7.35$ (3H, m, Ph), 7.36-7.42 (1H, m, Ph), 7.44-7.53 (3H, m, Ph), 8.07 (1H, dd, J 7.8, 0.8 Hz, Ph). ${ }^{13} \mathrm{C}$ NMR $\left(\mathrm{CDCl}_{3}\right): \delta_{\mathrm{C}} 20.5\left(\mathrm{q}, \mathrm{CH}_{3}\right), 20.6\left(\mathrm{q}, \mathrm{CH}_{3}\right), 25.4\left(\mathrm{t}, \underline{\mathrm{CH}_{2}} \mathrm{CH}_{2} \mathrm{C}\right), 26.0\left(\mathrm{~d}, \underline{\mathrm{CH}}\left(\mathrm{CH}_{3}\right)_{2}\right), 35.6(\mathrm{t}$, $\left.\mathrm{CH}_{2} \mathrm{CH}_{2} \mathrm{C}\right), 55.9$ (t, $\left.\mathrm{NCH}_{2}\right), 59.1\left(\mathrm{~s}, \mathrm{CH}_{2} \mathrm{CH}_{2} \underline{\mathrm{C}}\right), 62.1$ (t, $\left.\underline{\mathrm{CH}}_{2} \mathrm{CH}\left(\mathrm{CH}_{3}\right)_{2}\right), 75.3\left(\mathrm{t}, \mathrm{NCH}_{2} \mathrm{~N}\right), 126.9$, 127.0, 128.3, 128.4, 128.5, 129.9, 132.3, 133.5, 142.8, 144.6 (12C, Ph), 196.3 (s, C=O), 202.8 (s, $\mathrm{C}=\mathrm{S}$ ). Anal. Calcd. For $\mathrm{C}_{23} \mathrm{H}_{26} \mathrm{~N}_{2} \mathrm{OS}$ (378.53): C, 72.98; H, 6.92; N, 7.40; S, 8.47. Found: C, 72.40; H, 7.34; N, 7.30; S, 8.39\%.

1',3'-Dimethyl-4'-thioxo-2',3,3',4,4',6'-hexahydro-1H,1'H-spiro(naphthalene-2,5'-pyrimidin)-1-one (8). Yellowish powder, yield 82\%, mp 124-127 ${ }^{\circ} \mathrm{C}(\mathrm{EtOH})$; IR $\left(v_{\max }, \mathrm{cm}^{-1}\right): 1674$ $(\mathrm{C}=\mathrm{O}) .{ }^{1} \mathrm{H}$ NMR $\left(\mathrm{CDCl}_{3}\right): \delta_{\mathrm{H}} 2.25\left(1 \mathrm{H}, \mathrm{dt}, J 13.7,4.4 \mathrm{~Hz}, \mathrm{CH}_{2} \mathrm{C} \underline{\mathrm{H} H C}\right), 2.38\left(3 \mathrm{H}, \mathrm{s}, \mathrm{CH}_{3}\right), 2.83$ $\left(1 \mathrm{H}, \mathrm{d}, J 11.9 \mathrm{~Hz}, \mathrm{CHHCH}_{2} \mathrm{C}\right), 2.90-3.01(1 \mathrm{H}, \mathrm{m}, \mathrm{NC} \underline{H H}), 3.06\left(1 \mathrm{H}, \mathrm{d}, J 11.9 \mathrm{~Hz}, \mathrm{CH}^{-} \mathrm{HCH}_{2} \mathrm{C}\right)$, 3.09-3.19 (1H, m, NCHㅍ $), 3.21-3.33\left(1 \mathrm{H}, \mathrm{m}, \mathrm{CH}_{2} \mathrm{CH} \underline{\mathrm{HC}}\right), 3.43\left(3 \mathrm{H}, \mathrm{s}, \mathrm{CH}_{3}\right), 3.93-4.11(2 \mathrm{H}, \mathrm{m}$, $\left.\mathrm{NCH}_{2} \mathrm{~N}\right), 7.20-7.36(2 \mathrm{H}, \mathrm{m}, \mathrm{Ph}), 7.48(1 \mathrm{H}, \mathrm{t}, J 7.4 \mathrm{~Hz}, \mathrm{Ph}), 8.05(1 \mathrm{H}, \mathrm{d}, J 7.8 \mathrm{~Hz}, \mathrm{Ph}) .{ }^{13} \mathrm{C} \mathrm{NMR}$ $\left(\mathrm{CDCl}_{3}\right): \delta_{\mathrm{C}} 25.4\left(\mathrm{t}, \underline{\mathrm{CH}}_{2} \mathrm{CH}_{2} \mathrm{C}\right), 35.4\left(\mathrm{t}, \mathrm{CH}_{2} \underline{\mathrm{CH}}{ }_{2} \mathrm{C}\right), 41.0\left(\mathrm{q}, \mathrm{S}=\mathrm{CNCH}_{3}\right), 42.4\left(\mathrm{q}, \mathrm{CH}_{2} \mathrm{NCH}_{3}\right)$, $57.7\left(\mathrm{t}, \mathrm{N}-\mathrm{CH}_{2}\right), 59.3\left(\mathrm{~s}, \mathrm{CH}_{2} \mathrm{CH}_{2} \mathrm{C}\right), 74.5\left(\mathrm{t}, \mathrm{NCH}_{2} \mathrm{~N}\right), 126.9,128.4,128.5,132.0,133.5,142.7$ (6C, Ph), 196.3 (s, C=O), 199.5 (s, C=S). MS, m/z (\%) = $274(\mathrm{M}+, 76), 256$ (31), 241 (61) 200 (35), 184 (31), 155 (27), 128 (47), 116 (44), 90 (42), 58 (88), 42 (100). Anal. Calcd. For $\mathrm{C}_{15} \mathrm{H}_{18} \mathrm{~N}_{2} \mathrm{OS}$ (274.38): C, 65.66; H, 6.61; N, 10.21; S, 11.69. Found: C, 65.30; H, 6.88; N, 10.17 ; S, $11.98 \%$.

1'-Methyl-3'-phenyl-4' -thioxo-2',3,3',4,4',6'-hexahydro- $1 H, 1^{\prime} H$-spiro(naphthalene-2,5' pyrimidin)-1-one (9). White powder, yield 84\%; mp 130-132 ${ }^{\circ} \mathrm{C}(\mathrm{EtOH})$; IR $\left(v_{\max }, \mathrm{cm}^{-1}\right): 1674$ $(\mathrm{C}=\mathrm{O}) .{ }^{1} \mathrm{H}$ NMR $\left(\mathrm{CDCl}_{3}\right): \delta_{\mathrm{H}} 2.23-2.32\left(1 \mathrm{H}, \mathrm{m}, \mathrm{CH}_{2} \mathrm{CHHC}\right), 2.37\left(3 \mathrm{H}, \mathrm{s}, \mathrm{CH}_{3}\right), 2.82-2.99(2 \mathrm{H}$, $\left.\mathrm{m}, \mathrm{CHHCH}_{2} \mathrm{C}, \mathrm{NC} \underline{\mathrm{H}}\right), 3.00-3.14\left(1 \mathrm{H}, \mathrm{m}, \mathrm{CH}_{\underline{H C H}} \mathrm{C}\right), 3.19(1 \mathrm{H}, \mathrm{d}, J 12.2 \mathrm{~Hz}, \mathrm{NCH} \underline{\mathrm{H}}), 3.24-$ $3.38\left(1 \mathrm{H}, \mathrm{m}, \mathrm{CH}_{2} \mathrm{CH} \underline{\mathrm{HC}}\right), 4.10-4.25\left(2 \mathrm{H}, \mathrm{m}, \mathrm{NCH}_{2} \mathrm{~N}\right), 7.01-7.12(1 \mathrm{H}, \mathrm{m}, \mathrm{Ph}), 7.16(2 \mathrm{H}, \mathrm{d}, J 7.0$ $\mathrm{Hz}, \mathrm{Ph}), 7.23$ (2H, d, J 7.0 Hz, Ph), 7.26-7.33 (1H, m, Ph), 7.34-7.44 (2H, m, Ph), 8.00 (1H, d, $7.7 \mathrm{~Hz}, \mathrm{Ph}) .{ }^{13} \mathrm{C} \mathrm{NMR}\left(\mathrm{CDCl}_{3}\right): \delta_{\mathrm{C}} 25.4\left(\mathrm{t}, \underline{\mathrm{CH}}_{2} \mathrm{CH}_{2} \mathrm{C}\right), 35.7\left(\mathrm{t}, \mathrm{CH}_{2} \mathrm{CH}_{2} \mathrm{C}\right), 42.3$ (q, $\mathrm{S}=\mathrm{CNCH}_{3}$ ), $58.1\left(\mathrm{t}, \mathrm{N}-\mathrm{CH}_{2}\right), 59.0\left(\mathrm{~s}, \mathrm{CH}_{2} \mathrm{CH}_{2} \mathrm{C}\right), 76.2\left(\mathrm{t}, \mathrm{NCH}_{2} \mathrm{~N}\right), 127.0,128.3,128.4,128.5,128.6,129.1$, 130.0, 132.0, 133.6, 142.8, 144.7 (12C, Ph), 196.4 (s, C=O), 202.3 (s, C=S); Anal. Calcd. for $\mathrm{C}_{20} \mathrm{H}_{20} \mathrm{~N}_{2} \mathrm{OS}$ (336.45): C, 71.40; H, 5.99; N, 8.33; S, 9.53. Found: C, 71.13; H, 6.08; N, 8.29; S, $9.65 \%$.

3'-Phenyl-1'-(pyridin-3-ylmethyl)-4'-thioxo-2',3,3',4,4',6'-hexahydro-1H,1' $H$-spiro(naphthalene-2,5'-pyrimidin)-1-one (10). White powder, yield 79\%, mp 198-200 ${ }^{\circ} \mathrm{C}(\mathrm{EtOH})$; IR $\left(v_{\max }, \mathrm{cm}^{-1}\right): 1666(\mathrm{C}=\mathrm{O}) .{ }^{1} \mathrm{H} \mathrm{NMR}\left(\mathrm{CDCl}_{3}\right): \delta_{\mathrm{H}} 2.25-2.36\left(1 \mathrm{H}, \mathrm{m}, \mathrm{CH}_{2} \mathrm{C} \underline{\mathrm{H}} \mathrm{HC}\right), 2.73-2.87(1 \mathrm{H}$, $\left.\mathrm{m}, \mathrm{CHHCH}_{2} \mathrm{C}\right), 3.03-3.19$ (2H, m, $\left.\mathrm{CH}^{\mathrm{H}} \mathrm{HH}_{2} \mathrm{C}, \mathrm{NC} \underline{\mathrm{H}}\right)$, 3.22-3.36 (2H, m, $\left.\mathrm{CH}_{2} \mathrm{CH} \underline{\mathrm{HC}}, \mathrm{NCH} \underline{\mathrm{H}}\right)$, 3.77 (1H, d, J $13.6 \mathrm{~Hz}, \mathrm{C} \underline{H} H-P y r), 3.86$ (1H, d, J $13.6 \mathrm{~Hz}, \mathrm{CH} \underline{\mathrm{H}}-\mathrm{Pyr}), 4.37$ (1H, d, J $10.7 \mathrm{~Hz}$, NCㅂHN), $4.44(1 \mathrm{H}, \mathrm{d}, J 10.7 \mathrm{~Hz}, \mathrm{NCH} \underline{\mathrm{HN}}), 7.17(1 \mathrm{H}, \mathrm{d}, J 7.6 \mathrm{~Hz}, \mathrm{Ph}), 7.19-7.26$ (1H, m, Ph), 7.27-7.32 (3H, m, Ph, Pyr), 7.33-7.39 (1H, m, Ph), 7.41-7.50 (3H, m, Ph), 7.64-7.71 (1H, m, Pyr), 8.04 (1H, dd, J 7.9, $1.22 \mathrm{~Hz}, \mathrm{Ph}), 8.50(1 \mathrm{H}, \mathrm{dd}, J 4.8,1.6 \mathrm{~Hz}, \mathrm{Pyr}), 8.56(1 \mathrm{H}, \mathrm{s}, \mathrm{Pyr}) .{ }^{13} \mathrm{C}$ 
NMR $\left(\mathrm{CDCl}_{3}\right): \delta_{\mathrm{C}} 25.2\left(\mathrm{t}, \underline{\mathrm{CH}_{2}} \mathrm{CH}_{2} \mathrm{C}\right), 35.7\left(\mathrm{t}, \mathrm{CH}_{2} \underline{\mathrm{CH}_{2} \mathrm{C}}\right), 55.2\left(\mathrm{t}, \mathrm{CH}_{2}-\mathrm{Pyr}\right), 55.5\left(\mathrm{t}, \mathrm{NCH}_{2}\right)$, $58.9\left(\mathrm{~s}, \mathrm{CH}_{2} \mathrm{CH}_{2} \mathrm{C}\right), 74.5\left(\mathrm{t}, \mathrm{NCH}_{2} \mathrm{~N}\right), 123.6,127.0,128.3,128.4,128.6,130.0,132.0,132.1$, 133.6, 136.4, 142.7, 144.4, 149.4, 150.1 (17C, Ph, Pyr), 196.4 (s, C=O), 202.7 (s, C=S). Anal. Calcd. for $\mathrm{C}_{25} \mathrm{H}_{23} \mathrm{~N}_{3} \mathrm{OS}$ (413.53): C, 72.61; H, 5.61; N, 10.16; S, 7.75. Found: C, 72.72; H, 5.71; N, 10.27; S, 7.85\%.

(1-Benzyl-3-phenyl-4-thioxohexahydropyrimidin-5-yl)phenylmethanone (14). Yellowish powder, yield 88\%, mp 186-188 ${ }^{\circ} \mathrm{C}$; IR $\left(v_{\max }, \mathrm{cm}^{-1}\right)$ : 1660, $1672(\mathrm{C}=\mathrm{O}) .{ }^{1} \mathrm{H}$ NMR $\left(\mathrm{CDCl}_{3}\right): \delta_{\mathrm{H}}$ $3.39\left(3 \mathrm{H}, \mathrm{s}, \mathrm{CH}_{3}\right), 3.42\left(2 \mathrm{H}, \mathrm{s}, \mathrm{CH}_{2} \mathrm{~N}\right), 3.98\left(2 \mathrm{H}, \mathrm{s}, \mathrm{CH}_{2} \mathrm{Ph}\right), 4.46\left(2 \mathrm{H}, \mathrm{s}, \mathrm{NCH}_{2} \mathrm{~N}\right), 7.23-7.31$ $(1 \mathrm{H}, \mathrm{m}, \mathrm{Ph}), 7.34(2 \mathrm{H}, \mathrm{t}, J 7.2 \mathrm{~Hz}, \mathrm{Ph}), 7.41(2 \mathrm{H}, \mathrm{d}, J 7.2 \mathrm{~Hz}, \mathrm{Ph}), 7.77-7.88(2 \mathrm{H}, \mathrm{m}, \mathrm{Ph}), 7.96-$ $8.08(2 \mathrm{H}, \mathrm{m}, \mathrm{Ph}) .{ }^{13} \mathrm{C} \mathrm{NMR}\left(\mathrm{CDCl}_{3}\right): \delta_{\mathrm{C}} 40.6\left(\mathrm{q}, \mathrm{CH}_{3}\right), 53.2\left(\mathrm{t}, \mathrm{NCH}_{2}\right), 56.9\left(\mathrm{t}, \mathrm{CH}_{2} \mathrm{Ph}\right), 65.7(2$, C-C $=\mathrm{S}), 71.6\left(\mathrm{t}, \mathrm{NCH}_{2} \mathrm{~N}\right), 124.3,127.8,128.6,129.1,135.7,136.9,143.4(12 \mathrm{C}, \mathrm{Ph}), 190.1$ (s, $\mathrm{C}=\mathrm{O}$ ), 197.1 (s, $\mathrm{C}=\mathrm{S}$ ). Anal. Calcd. for $\mathrm{C}_{24} \mathrm{H}_{22} \mathrm{~N}_{2} \mathrm{OS}$ (386.15): C, 74.58; H, 5.74; N, 7.25; S, 8.30. Found: C, 74.33; H, 5.69; N, 7.18; S, 8.26\%.

Synthesis of $\mathbf{1}^{\prime}$-benzyl-3'-methyl-4'-thioxo-2', $3^{\prime}, 4^{\prime}, 6^{\prime}$-tetrahydro-1'H-spiro(indene-2,5'pyrimidine)-1,3-dione (12). To a solution of benzylamine ( $3 \mathrm{mmol}, 0.32 \mathrm{~g}$ ) and formaldehyde ( $6 \mathrm{mmol} ; 0.49 \mathrm{~g}$, a $37 \%$ solution in water) in $15 \mathrm{~mL}$ of EtOH, 1,3-dioxoindane-2-carbothioic $N$ methylamide ( $1 \mathrm{mmol}, 0,219 \mathrm{~g}) \mathbf{1 1}$ and $1 \mathrm{~mL}$ of acetic acid as catalyst were added under stirring. The reaction mixture was stirred at room temperature for 1 hour and then left for 2-3 days. The crude crystals were separated by filtration and purified by crystallization from EtOH. The product formed as a white powder, yield $65 \%, \mathrm{mp} 143-145{ }^{\circ} \mathrm{C}$; IR $\left(v_{\max }, \mathrm{cm}^{-1}\right): 1666,1702$ $(\mathrm{C}=\mathrm{O}) .{ }^{1} \mathrm{H}$ NMR $\left(\mathrm{CDCl}_{3}\right): \delta_{\mathrm{H}} 3.29-3.42(1 \mathrm{H}, \mathrm{m}, \mathrm{C} \underline{\mathrm{H} H C H}), 3.54(1 \mathrm{H}, \mathrm{dd}, J 13.5,8.9 \mathrm{~Hz}$, $\mathrm{CH} \underline{\mathrm{HCH}}), 4.00\left(2 \mathrm{H}, \mathrm{dd}, J 20.0,13.3 \mathrm{~Hz}, \mathrm{CH}_{2} \mathrm{Ph}\right), 4.37(1 \mathrm{H}, \mathrm{dd}, J 12.3,1.9 \mathrm{~Hz}, \mathrm{NC} \underline{H} \mathrm{HN}), 4.80$ $(1 \mathrm{H}, \mathrm{d}, J 12.5 \mathrm{~Hz}, \mathrm{CHC}=\mathrm{O}), 5.28-5.41(1 \mathrm{H}, \mathrm{m}, \mathrm{NCH} \underline{\mathrm{HN}}), 7.23-7.32(3 \mathrm{H}, \mathrm{m}, \mathrm{Ph}), 7.33-7.42(5 \mathrm{H}$, $\mathrm{m}, \mathrm{Ph}), 7.43-7.52(4 \mathrm{H}, \mathrm{m}, \mathrm{Ph}), 7.54-7.62(1 \mathrm{H}, \mathrm{m}, \mathrm{Ph}), 8.01-8.10(2 \mathrm{H}, \mathrm{m}, \mathrm{Ph}) .{ }^{13} \mathrm{C} \mathrm{NMR}\left(\mathrm{CDCl}_{3}\right)$ : $\delta_{\mathrm{C}} 51.7\left(\mathrm{t}, \mathrm{NCH}_{2}\right), 52.8(\mathrm{~d}, \underline{\mathrm{CH}}-\mathrm{C}=\mathrm{S}), 56.8\left(\mathrm{t}, \mathrm{CH}_{2} \mathrm{Ph}\right), 73.7\left(\mathrm{t}, \mathrm{NCH}_{2} \mathrm{~N}\right), 126.7,128.0,128.3$, 128.7, 128.8, 129.3, 129.9, 133.5, 136.6, 136.8, 144.0 (18C, Ph), 196.7 (s, C=O), 197.9 (s, C=S); Anal. Calcd. for $\mathrm{C}_{20} \mathrm{H}_{18} \mathrm{~N}_{2} \mathrm{O}_{2} \mathrm{~S}$ (350.43): C, 68.55; H, 5.18; N, 7.99; S, 9.15. Found: C, 68.28; H, 5.28; N, 7.91; S, 9.35\%.

\section{Acknowledgements}

We express our thanks to Professor Jerzy Lange of the Warsaw University of Technology for helpful discussions and assistance with the manuscript. 


\section{References}

1. Jagodziński, T. S. Chem. Rev. 2003, 103, 197. http://dx.doi.org/10.1021/cr0200015 PMid:12517184

2. Britsun, V. N.; Esipenko, A. N.; Lozinskii, M. O. Chem. Heterocycl. Comp. 2008, 44, 1429. http://dx.doi.org/10.1007/s10593-009-0214-x

3. Wesołowska, A.; Groś, Ł.; Westerlich, S.; Jagodzinski, T. S. Arkivoc 2008, (xv), 239. http://dx.doi.org/10.3998/ark.5550190.0009.f21

4. Belskaya, N.P.; Dehaen, W.; Bakulev, V. Arkivoc 2010, (i), 275. http://dx.doi.org/10.3998/ark.5550190.0011.108

5. Zhang, X.; Teo, S. W.-T.; Chan, P. W.-H. J. Org. Chem. 2010, 75, 6290. http://dx.doi.org/10.1021/jo101292r PMid:20795628

6. Zaleska, B.; Karelus, M.; Flasiński, M.; Serda, P. Arkivoc 2007, (vi), 64.

7. Bogdanowicz-Szwed, K.; Grochowski, J.; Obara, A.; Rys, B.; Serda, P. J. Org. Chem. 2001, $66,7205$.

http://dx.doi.org/10.1021/jo010349k

8. Bogdanowicz-Szwed, K.; Budzowski, A.; Gil, R.; Serda, P. Monatsh. Chem. 2010, 141, 63. http://dx.doi.org/10.1007/s00706-009-0233-4

9. Groszkowski, S.; Korzycka, L.; Białasiewicz, W. Pol. J. Pharmacol. Pharm. 1973, 25, 573. http://dx.doi.org/10.1111/j.2042-7158.1973.tb09160.x PMid:4792477

10. Drandarov, K.; Guggisberg, A.; Hesse, M. Helv. Chim. Acta 1999, 82, 229. http://dx.doi.org/10.1002/(SICI)1522-2675(19990210)82:2<229::AID-HLCA229>3.0.CO;2$\mathrm{H}$

11. Siddiqui, A. Q.; Merson-Davies, L.; Cullis, P. M. J. Chem. Soc., Perkin Trans. 1 1999, 3243.

12. Hrvath, D. J. J. Med. Chem. 1999, 40, 2412 and references therein. http://dx.doi.org/10.1021/jm9603781 PMid:9240356

13. Beresnevičius, Z. J.; Mickievičius, V.; Rutkauskas, K.; Kantminienë, K. Pol. J. Chem. Tech. 2003, 5,75 .

14. Chantrapromma, K.; Ganem, B. Tetrahedron Lett. 1981, 22, 23. http://dx.doi.org/10.1016/0040-4039(81)80030-5

15. Mayr, M.; Buchmeister, M. R. Macromol. Rapid Commun. 2004, 25, 231. http://dx.doi.org/10.1002/marc.200300173

16. Mayr, M.; Wurst, K.; Ongania, K.-H.; Buchmester, M. R. Chem.-Eur. J. 2004, 10, 1256. http://dx.doi.org/10.1002/chem.200305437 PMid:15007815

17. Perillo, I. A.; Garcia, M. B.; Bisceglia, J. A.; Orelli, L. R.; J. Heterocycl. Chem. 2002, 39, 655. http://dx.doi.org/10.1002/jhet.5570390409

18. Katritzky, A. R.; Singh, S. K.; He, H.-Y. J. Org. Chem. 2002, 67, 3115. http://dx.doi.org/10.1021/jo010927x

19. Wei H.-L.; Yan, Z.-Y.; Niu, Y.-N.; Li, G.-Q.; Liang Y.-M. J. Org. Chem. 2007, 72, 8600. 
http://dx.doi.org/10.1021/jo7016235 PMid:17918899

20. Kleinmann, E. F. In Comprehensive Organic Synthesis; Trost, B. M., Ed., Pergamon Press: New York, 1991, vol.2, chapter 4.1.

21. Kobayashi, S.; Ishitani, H. Chem. Rev. 1999, 99, 1069. http://dx.doi.org/10.1021/cr980414z PMid:11749440

22. Córdova, A. Acc. Chem. Res. 2004, 37, 102. http://dx.doi.org/10.1021/ar0302311 PMid:14967057

23. Marques, M. M. B. Angew. Chem., Int. Ed. 2006, 45, 348. http://dx.doi.org/10.1002/anie.200502630 PMid:16342308

24. Ramón, D. J.; Yus, M. Angew. Chem., Int. Ed. 2005, 44, 1602. http://dx.doi.org/10.1002/anie.200460548 PMid:15719349

25. Chanda, A.; Fokin, V. V. Chem. Rev. 2009, 109, 725. http://dx.doi.org/10.1021/cr800448q PMid:19209944

26. 26. Zhou, L.; Bohle, D. S.; Jiang, H. F.; Li, C. J. Synlett 2009, 75.

27. Mukhopadhyay, C.; Rana, S.; Butcher, R. J. Tetrahedron Lett. 2011, 52, 4153 and references therein. http://dx.doi.org/10.1016/j.tetlet.2011.05.144

28. Shah, T. B.; Gupte, A.; Patel, M. R.; Chaudhari, V. S.; Patel, H.; Patel, V.C. Indian. J. Chem. Sec. B. 2010, 49B, 578.

29. Wesołowska, A.; Jagodziński, T. S.; Sośnicki, J. G. Polish J. Chem. 2001, 75, 387.

30. Jagodziński, T. S.; Wesołowska, A.; Sośnicki, G. J. Polish J. Chem. 2000, 74, 1101.

31. Jagodziński, T. S.; Sośnicki J. G.; Wesołowska A. Tetrahedron 2003, 53, 4183. http://dx.doi.org/10.1016/S0040-4020(03)00576-3

32. Bernát, J.; Kristian, P.; Guspanová, J.; Chomca, I. Heterocycles 2000, 53, 1171. http://dx.doi.org/10.3987/COM-00-8845

33. Bogdanowicz-Szwed, K.; Nowak, I.; Tyrka, M. J. Prakt. Chem. / Chem.- Ztg. 1975, 337, 71. http://dx.doi.org/10.1002/prac.19953370117. 